\section{REVISTA BRASILEIRA DE QUALIDADE DE VIDA}

\title{
Grau de concordância do IMC e do IAC com percentual de gordura corporal
}

\section{Degree of agreement with BAI and BMI body fat percentage}

\author{
Rodrigo Gonçalves \\ Universidade do Contestado - UnC - Porto União - Santa Catarina - Brasil \\ rodrigo.1312@hotmail.com \\ Luis Paulo Gomes Mascarenhas \\ Universidade Estadual do Centro-Oeste - Unicentro - Irati - Paraná - Brasil \\ masca58@hotmail.com \\ Edna Caroline Liebl \\ Universidade do Contestado - UnC - Porto União - Santa Catarina - Brasil \\ ecliebl@hotmail.com \\ Valderi Abreu de Lima \\ Universidade do Contestado - UnC - Porto União - Santa Catarina - Brasil \\ valderiabreulima@hotmail.com \\ Wallace Bruno de Souza \\ Universidade do Vale do Itajaí - Univali - Itajaí - Santa Catarina - Brasil \\ wallace.bsouza@yahoo.com.br \\ Marcos Tadeu Grzelczak \\ Universidade do Contestado - UnC - Porto União - Santa Catarina - Brasil \\ marcosacupuntura@ig.com.br \\ William Cordeiro de Souza \\ Universidade do Contestado - UnC - Porto União - Santa Catarina - Brasil \\ professor_williamsouza@yahoo.com.br
}

\section{RESUMO}

OBJETIVO: Verificar qual método indireto apresenta maior concordância e eficácia na identificação de excesso de gordura corporal: o Índice de Massa Corporal (IMC) ou o Índice de Adiposidade Corporal (IAC).

MÉTODOS: A amostra foi constituída de 20 voluntários, com idades entre 19 e 49 anos. O nível de atividade física foi avaliado pelo IPAQ. Foram aferidos o percentual de gordura corporal através de Dobras Cutâneas, peso e altura para o cálculo do IMC e circunferência do quadril para o cálculo do IAC. Para análise dos dados foi o método de Bland Altman com nível de significância $\mathrm{p}<0,05$.

RESULTADOS: Observou-se que o IMC teve uma correlação inexistente com percentual de gordura $(\mathrm{r}=-0,027, \mathrm{p}=0,907)$ e, em contrapartida, o IAC apresentou uma forte correlação com o percentual de gordura $(\mathrm{r}=0,816, \mathrm{p}<0,001)$. É valido ressaltar que quando confrontado o IMC e o IAC obteve-se uma correlação fraca $(\mathrm{r}=0,408, \mathrm{p}=0,073)$.

CONCLUSÕES: O IAC apresentou forte relação com a gordura corporal, podendo ser um indicador aplicável. 
PALAVRAS-CHAVE: Índice de Massa Corporal. Índice de Adiposidade Corporal. Dobras Cutâneas. Composição Corporal.

\begin{abstract}
OBJECTIVE: To determine which indirect method shows greater agreement and effectiveness in identifying excess body fat: the Body Mass Index (BMI) or Body Adiposity Index (BAI).

METHODS: The sample consisted of 20 volunteers, aged 19 to 49 years. The level of physical activity was assessed by IPAQ. The percentage of body fat was measured by Skin Folds, weight and height to calculate BMI and hip circumference to calculate the BAI. Data analysis was the Bland Altman method with significance level $\mathrm{p}<0.05$.

RESULTS: It was observed that BMI had a non-existent with fat percentage $(r=-0.027, p=0.907)$ correlation and in return, the BAI had a strong correlation with body fat percentage $(\mathrm{r}=0.816, \mathrm{p}<$ $0.001)$. It is worth noting that when confronted BMI and BAI, gave a weak correlation $(r=0.408$, $\mathrm{p}=0.073$ ).
\end{abstract}

CONCLUSIONS: The BAI had strong relationship with body fat, may be a relevant indicator.

KEYWORDS: Body Mass Index. Body Adiposity Index. Skin Folds. Body Composition.

\title{
1. Introdução
}

Diversas técnicas para avaliação da composição corporal vêm sendo desenvolvidas, porém, muitas destas apresentam um alto custo financeiro na sua realização. Torna-se, portanto, necessário o desenvolvimento de técnicas simples, mais baratas e com boa precisão, para aplicação no campo e em grandes populações (POMPEU et al., 2004).

Por ser de baixo custo operacional e de uma grande facilidade na coleta de dados, pois sua fórmula necessita apenas do peso $(\mathrm{kg})$ e da estatura $(\mathrm{m})$ do avaliado, o índice de massa corporal (IMC) é altamente utilizado. Porém, afirmar que um indivíduo está acima do peso, fundamentando-se apenas em um valor de massa corporal e da estatura se traduz em uma ação invasiva, pois, como afirma Fernandes (2003), o IMC não leva em conta a composição corporal proporcional do organismo como a massa óssea e a massa magra ou gorda.

Devido o IMC ser um método muito utilizado, porém pouco confiável e dependente das especificidades do avaliado, como por exemplo, um atleta, na maioria dos casos o mesmo manifesta baixo nível de gordura corporal e um alto nível de massa magra, o que resulta em um IMC acima do desejável, por conta da massa magra e não do excesso de gordura.

Em 2011, nos Estados Unidos, Bergman e colaboradores realizaram uma pesquisa com Africano-Americanos tentando determinar a adiposidade com uma equação básica, chamada de Índice de Adiposidade Corporal (IAC), fácil de ser aplicada, com um gasto de tempo pequeno e que fosse mais precisamente aceitável que o próprio IMC. Utilizando-se das medidas de circunferência do quadril e a altura, esse novo método pode ser utilizado para refletir o percentual de gordura corporal tanto para homens adultos quanto para mulheres de diferentes etnias, sem correção numérica. Para estruturação dessa nova fórmula foi usado o instrumento DEXA na avaliação do \%G, considerado padrão ouro. Encontrou-se uma forte correlação entre os parâmetros do IAC (BERGMAN et al., 2011).

Souza et al. (2013) correlacionaram o IAC com o IMC e verificaram que houve uma correlação positiva e significativa entre o IAC e o IMC, em atletas profissionais de futebol, concluindo que o IAC pode ser uma ferramenta favorável, na determinação da adiposidade corporal.

O presente estudo teve como objetivo verificar qual método apresenta maior concordância e eficácia na identificação da gordura corporal: o IMC ou o IAC. 


\section{Métodos}

A amostra intencional foi constituída de 14 homens com idades entre 19 e 44 anos e 6 mulheres com idades entre 19 e 49 anos, todos acadêmicos da Universidade do Contestado - Canoinhas - SC.

Foi utilizado a versão curta do Questionário Internacional de Atividade Física (IPAQ) para mensuração do nível de atividade física. Este instrumento contém quatro questões que analisam a participação em atividades físicas moderadas e vigorosas dos respondentes durante a última semana (MATSUDO et al., 2001). O IPAQ também fornece dados como a estimativa do tempo semanal gasto em atividades físicas de intensidade moderada e vigorosa, em diferentes contextos do cotidiano, e, ainda, o tempo despendido em atividades passivas, realizadas na posição sentada (BENEDETTI et al., 2007).

Foram utilizados, também, uma balança e um estadiômetro mecânico antropométrico da marca Welmy, um adipômetro da marca Cescorf Fww860 Mitutoyo, com precisão de 0,1mm, uma trena antropométrica da marca Sanny Medical 2 mt modelo SN-4011.

$\mathrm{Na}$ aferição da estatura, o avaliado estava na posição ortostática (em pé), pés unidos, colocando em contato com o instrumento de medida as superfícies posteriores do calcanhar. A medida foi feita com o indivíduo em apneia inspiratória, de modo a minimizar possíveis variações sobre esta variável antropométrica. A cabeça estava orientada no plano de Frankfurt, paralela ao solo. A medida foi feita com o cursor em ângulo de 90 graus em relação à escala. Permitiu-se ao avaliado usar calção e camiseta (sendo estas roupas leves), exigindo-se que estivesse descalço. A aferição da massa corporal total aconteceu com o avaliado posicionado em pé, de costas para a escala da balança, com afastamento lateral dos pés. Assim, com estes dados foi calculado o IMC (DELGADO, 2004).

No cálculo do \%G seguiu-se os procedimentos de Delgado (2004). Primeiramente, realizouse a aferição da dobra cutânea Tricipital, onde a referencia anatômica foi o processo acromial da escapula e o processo olecraniano da ulna. Com o indivíduo em pé e braços relaxados ao longo do corpo foi medida a dobra na face posterior do braço, na distância média entre a borda superolateral do acrômio e o bordo inferior do olecrano. Sua determinação foi realizada seguindo o eixo longitudinal do membro. Para a aferição da dobra cutânea supra-ilíaca, sua referencia anatômica foi a crista ilíaca. Metade da distância entre o último arco costal e a crista ilíaca, sobre a linha axilar medial. Era necessário que o avaliado afastasse o braço para trás para permitir a execução da medida.

Na determinação da dobra cutânea da coxa-medial a referencia anatômica foi a linha inguinal e a patela. A medida foi feita sobre o músculo reto femoral, a um terço da distância do ligamento inguinal e a borda superior da patela, em conformidade com procedimento sugerido por Guedes (1985), e na metade desta distância sugerida por Pollock e Wilmore (1993). Já a dobra cutânea torácica foi aferida tendo como referencia anatômica axila e mamilo. A medida foi feita na metade da distância entre a linha axilar anterior e o mamilo, para homens, e a um terço da linha axilar anterior, para mulheres. Por fim, a medida da dobra cutânea abdominal teve como ponto de referencia a cicatriz umbilical. Foi medido aproximadamente de $3 \mathrm{~cm}$ a $1 \mathrm{~cm}$ à direita desta cicatriz.

Para o cálculo do \%G foram utilizadas as equações sugeridas por Jackson e Pollock (1978).

A circunferência do quadril é a extensão posterior máxima dos glúteos. Tomada ao nível dos pontos trocantéricos direito e esquerdo. Deve ser realizada paralelamente ao solo, estando o avaliado com os pés unidos (DELGADO, 2004).

O cálculo do IAC foi realizado dividindo-se a medida da circunferência do quadril $(\mathrm{cm})$, pela altura $(\mathrm{m})$, multiplicada pela raiz quadrada da altura $(\mathrm{m})$, e diminui-se 18 do resultado final (BERGMAN et al., 2011).

É válido ressaltar que todas as coletas de dados aconteceram no núcleo de estudos da atividade física (NEAF), sempre no período da manhã. Todos os avaliados preencheram um termo de consentimento livre e esclarecido. Este trabalho foi aprovado pelo Comitê de Ética da Plataforma Brasil, da Universidade do Contestado - UnC (número do Parecer: 62044). 
Para o tratamento estatístico foi utilizado o software Med Calc, aonde se calculou a correlação de Pearson, e o método de Bland Altman para determinação do grau de concordância entre o IMC, o IAC e o \%G com nível de significância estipulado em $p<0,05$.

\section{Resultados}

Na Tabela 1 estão apresentadas variáveis antropométricas dos avaliados para a caracterização da amostra estudada:

Tabela 1 - Caracterização da amostra

\begin{tabular}{c|cc}
\hline & $\begin{array}{c}\text { Média } \\
(\mathbf{n = 2 0})\end{array}$ & $\begin{array}{c}\text { Desvio } \\
\text { Padrão }\end{array}$ \\
\hline Idade & 23 & $\pm 8,34$ \\
Peso (Kg) & 68,4 & $\pm 11,5$ \\
Altura (m) & 1,73 & $\pm 0,09$ \\
IMC & 23,9 & $\pm 2,82$ \\
Circunferência Quadril (CQ) & 0,94 & $\pm 0,05$ \\
IAC & 23,4 & $\pm 4,49$ \\
\%G & 13,2 & $\pm 8,66$ \\
\hline
\end{tabular}

Fonte: Autoria própria (2014).

A amostra foi composta por indivíduos classificados através do questionário IPAQ, sendo 50\% destes ativos, $20 \%$ muito ativos e $30 \%$ sedentários. Os resultados encontrados nas correlações realizadas entre as variáveis avaliadas podem ser verificados na Tabela 2:

Tabela 2 - Correlação entre as variáveis avaliadas

\begin{tabular}{c|cc}
\hline & $\mathbf{r}$ & $\boldsymbol{P}$ \\
\hline \%G x IMC & $-0,027$ & 0,907 \\
\%G x IAC & 0,816 & $<0,001^{*}$ \\
IMC x IAC & 0,408 & 0,073 \\
\hline
\end{tabular}

Fonte: Autoria própria (2014).

$$
\text { Obs.: } * \mathrm{p}<0,05
$$

Obteve-se então uma correlação significativa entre as dobras cutâneas (DC) e o IAC e uma ausência de significância entre as DC e o IMC e entre o IMC e o IAC. Nos Gráficos 1,2 e 3 estão apresentados através de diagramas de dispersão, os valores correlacionais encontrados.

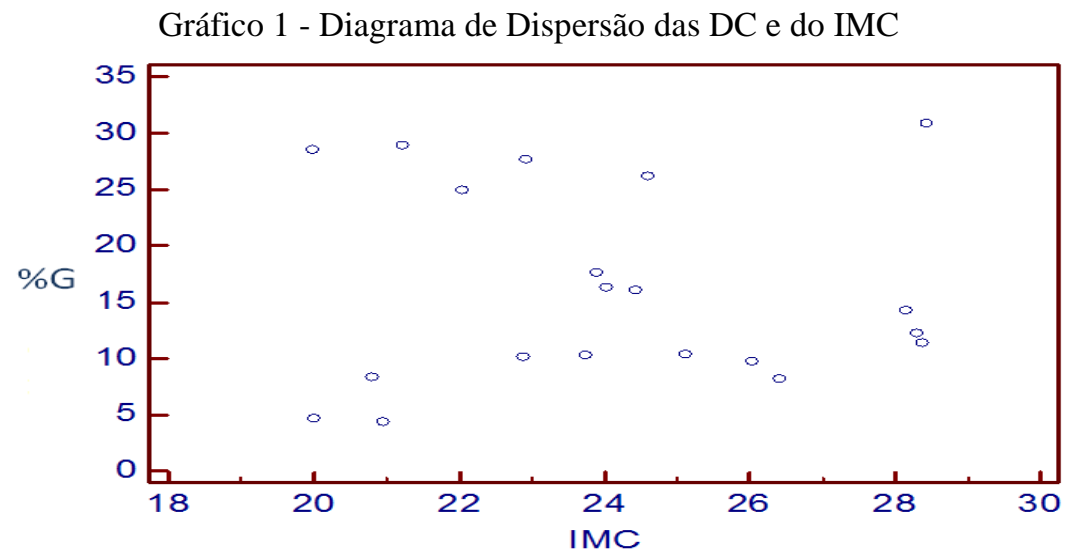

Fonte: Autoria própria (2014) 
Gráfico 2 - Diagrama de Dispersão das DC e do IAC

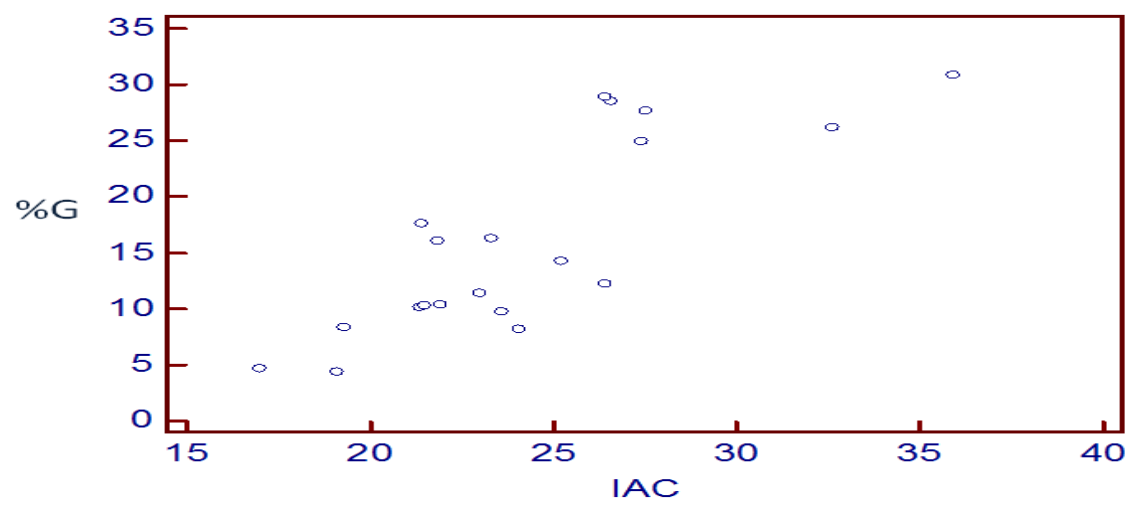

Fonte: Autoria própria (2014).

Gráfico 3 - Diagrama de Dispersão do IAC e do IMC

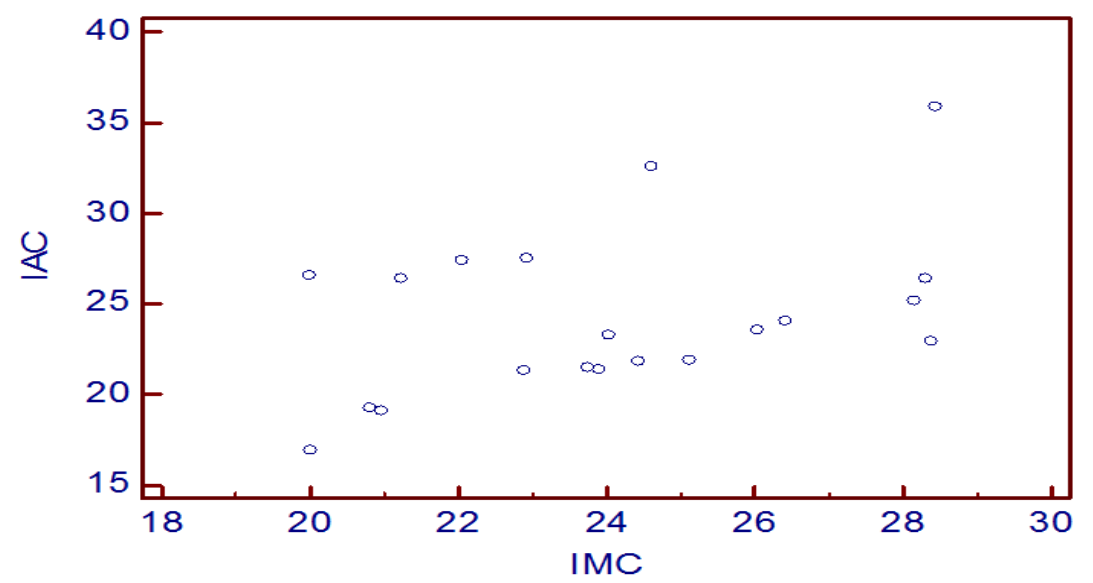

Fonte: Autoria própria (2014).

Os Gráficos 1, 2 e 3 apresentam grandes distinções entre si. Nos Gráficos 1 e 3 visualizam-se pontos dispersos, retratando uma correlação inexistente. Já no Gráfico 2 podem-se observar pontos orientados em uma reta ascendente, caracterizando uma correlação forte.

No esforço para ilustrar a concordância das DC com o IMC, das DC com o IAC e do IMC com o IAC, foi usado o método Bland-Altman (Gráficos 4, 5 e 6). Esse método ajuda prover informações úteis no que diz respeito às faixas de valores para mostrar se as variáveis avaliadas são concordantes/discordantes (BERGMAN et al., 2011).

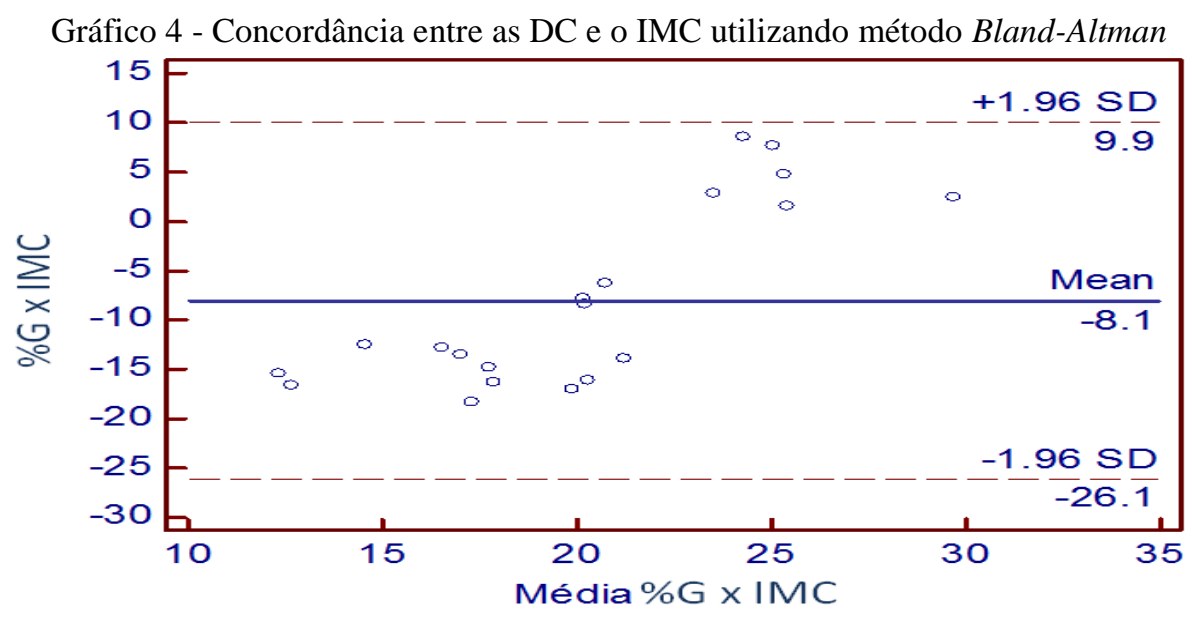

Fonte: Autoria própria (2014). 
Quanto mais perto os pontos se dispuserem ao longo de uma reta, maior será o grau de associação entre as duas variáveis. No Gráfico 4 os pontos estão muito dispersos, indicando uma correlação inexistente entre as DC e o IMC.

Gráfico 5 - Correlação entre as DC e o IAC, utilizando o método Bland-Altman

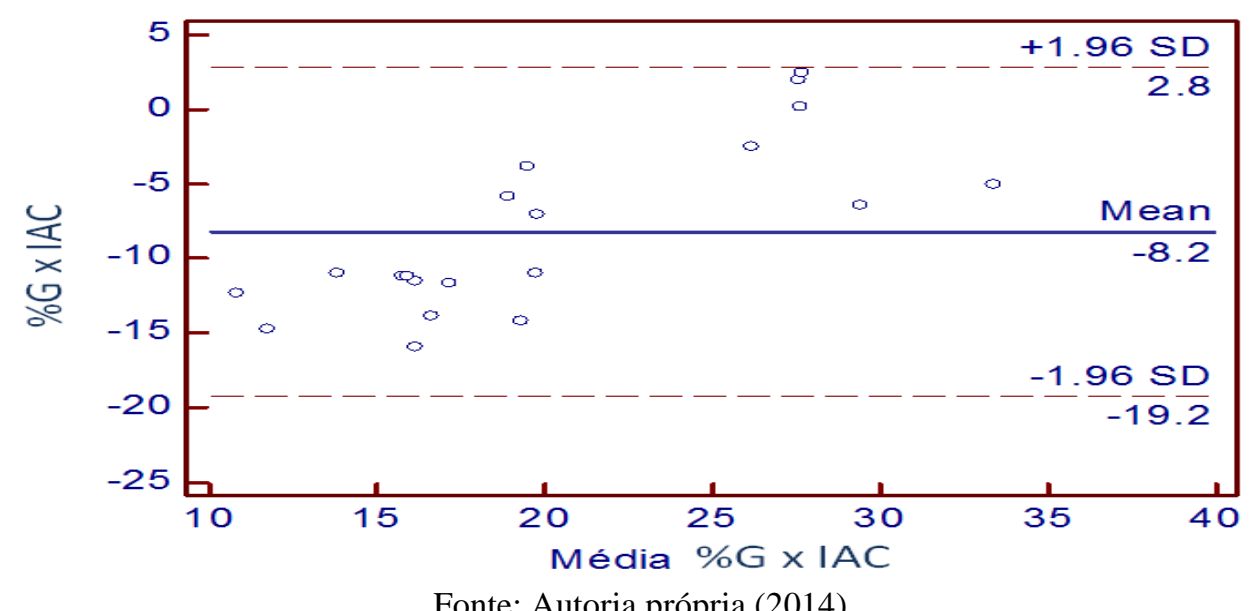

Já no Gráfico 5 os pontos orientados indicam uma correlação positiva entre o \%G e o IAC. O Gráfico 6 ilustra uma fraca correlação entre o IMC e o IAC, observando um grande afastamento dos pontos em relação à reta.

Gráfico 6 - Correlação entre o IMC e o IAC, utilizando o método Bland-Altman

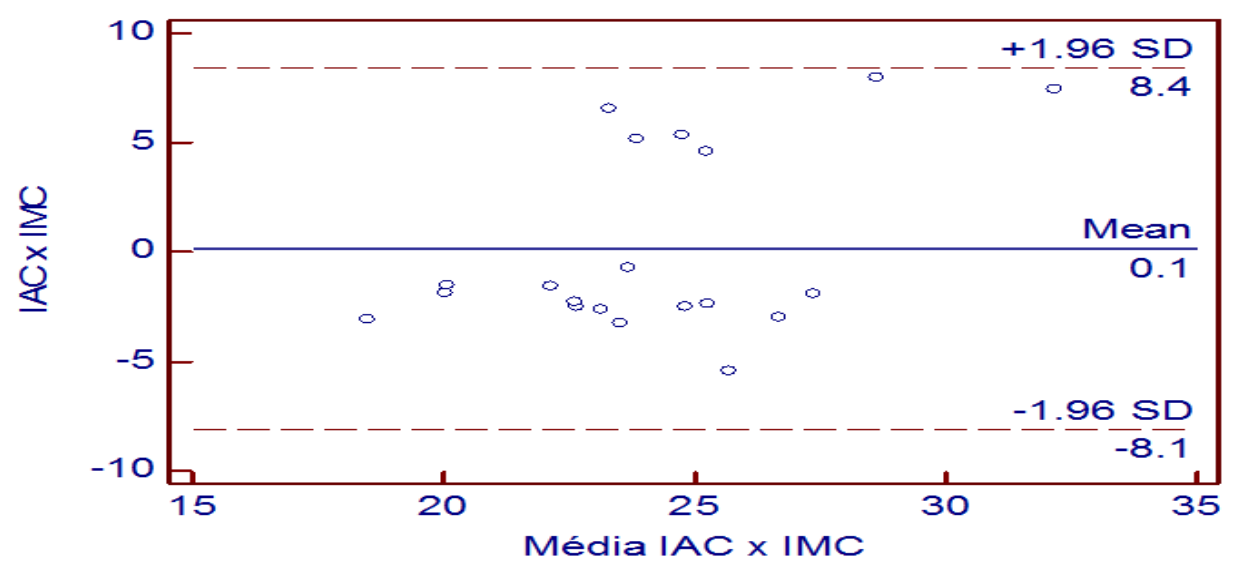

Fonte: Autoria própria (2014).

\section{Discussão}

Depois do estudo de validação de Bergman et al. (2011), o IAC passou a ser visto como um bom e útil mecanismo para avaliação da saúde, podendo substituir outros índices de adiposidade corporal, incluindo o próprio IMC. Corroborando com os achados deste estudo, onde também foi verificada uma forte correlação $(r=0,81, \mathrm{p}<0,001)$ do IAC com a composição corporal.

Em estudo realizado por Sulino et al. (2011), com o objetivo de comparar o IAC com as DC, foram avaliados 308 indivíduos, com idades entre 18 e 65 anos. Foi concluído que o IAC pode ser utilizado de forma satisfatória para estimar o $\% \mathrm{G}$, devido a sua forte relação com os resultados das DC, sendo que, neste caso, a correlação entre o IAC e as DC foi de $r=0,73$ com $p<0,01$ para homens e $\mathrm{r}=0,70 \mathrm{com} p<0,01$ para mulheres. Obtendo, então, um índice bem próximo aos encontrados nessa pesquisa. É válido ressaltar que este estudo separou os indivíduos por sexo, diferentemente deste trabalho, podendo isso ser uma variável que interferiu nos resultados. 
Januário et al. (2008), em estudo comparando o IMC e as DC, com uma amostra de escolares de 8 a 10 anos de ambos os sexos, detectou uma concordância moderada entre os indicadores de obesidade na classificação para meninos e meninas (Kappa $=0,43$ e 0,50, respectivamente). Os dados dessa pesquisa demonstraram que $21 \%$ dos meninos e $15 \%$ das meninas se encontravam em uma faixa de peso considerada normal, segundo o IMC, porém, apresentavam um quadro de obesidade quando avaliado o \%G por DC. Observa-se, assim, certa inconsistência no método do IMC em classificar crianças.

Lópes et al. (2012) avaliaram indivíduos com idades médias de 39,2 anos, caucasianos, de uma zona Mediterrânica e Europeia, com o objetivo de comparar os métodos do IAC, do IMC e do $\% \mathrm{G}$ através da bioimpedância. Foram verificadas as seguintes correlações: entre o IAC e o IMC $(\mathrm{r}=0,64, \mathrm{p}=0,001)$, entre o IAC e o $\% \mathrm{G}(\mathrm{r}=0,74, \mathrm{p}=0,001)$ e entre o IMC e o $\% \mathrm{G}$ a $(\mathrm{r}=0,54, \mathrm{p}=0,001)$. Os autores concluíram que o IAC poderia ser uma ferramenta tão fidedigna para medir a adiposidade quanto os equipamentos elétricos ou complexos sistemas mecânicos.

Com o objetivo de comparar os métodos para estimar o \%G, o IAC e o IMC e a relação cintura quadril, em incidentes Diabéticos tipo II, Schulze et al. (2012) constataram que o IMC foi maior correlacionado com o $\% \mathrm{G}$ (homens $\mathrm{r}=0,81$ e mulheres $\mathrm{r}=0,84)$ do que o IAC $(\mathrm{r}=0,68$ e 0,81 ), enquanto que a relação cintura/quadril mostrou as correlações mais fortes (homens $r=0,84$, mulheres $\mathrm{r}=0,88$ ). Foi observado, ainda, que a relação cintura quadril é um melhor preditor do $\% \mathrm{G}$ do que o IAC e o IMC. Esses resultados contrapõem os achados do presente estudo.

Johnson et al. (2012) correlacionaram o IAC e o IMC com o \%G, via DEXA, em Europeuamericanos, adultos, encontrando os seguintes resultados: entre o IAC e o \%G via DEXA $(r=0,752)$ e entre o IMC e o \%G via DEXA $(\mathrm{r}=0,445)$. As conclusões desse estudo mostraram que o IAC fornece um melhor indicador da adiposidade do que o IMC, corroborando com os achados deste estudo.

Freedman et al. (2013) fizeram uma crítica ao método do IAC, já que, no seu estudo, todas as análises indicaram que o mesmo teve baixa associação com as pregas cutâneas, os fatores de risco cardiovascular, a circunferência da cintura e o IMC. Um índice global de sete fatores de risco cardiovascular teve maior correlação com o IMC $(r=0,58)$ e circunferência da cintura $(r=0,61)$ do que com o IAC $(r=0,49)$. Esse estudo indica que, provavelmente, o IAC é um índice de adiposidade inferior do que o IMC e da circunferência da cintura.

Lemacks et al. (2012) buscaram verificar se o IAC poderia ser validado em mulheres pósmenopáusicas obesas, a fim de substituir a utilização do IMC, para determinar o excesso de gordura corporal. O coeficiente de concordância revelou uma concordância ruim, não havendo diferença entre os métodos do IAC e do IMC. Sendo assim, com base nos resultados deste estudo, o IAC tem limitações para uso em mulheres que estão na pós-menopausa e obesas, mas pode ser prático para aplicações de pesquisa e, eventualmente, serve como um método fácil para estimar sobrepeso e obesidade em outros ambientes.

Esco (2012) verificou a precisão do IAC para prever o \% em mulheres atletas colegiais utilizando DEXA como a variável critério. No entanto, o IAC não forneceu uma correlação significativa com o DEXA $\left(r=0,28, \mathrm{R}^{2}=0,08, \mathrm{p}>0,05\right)$. Os resultados desta investigação indicam que o IAC possui erros individuais na previsão do $\% \mathrm{G}$ em atletas do sexo feminino e tem uma tendência a fornecer valores superestimados quando o \%G diminui. Portanto, este método não pode ser usado para predizer o \% $\mathrm{G}$ em mulheres atléticas.

\section{Conclusão}

No presente estudo constatou-se que o IAC apresentou forte correlação com as DC $(\mathrm{r}=0,816$ $\mathrm{p}<0.001)$. Além disso, verificou uma correlação inexistente quando comparados os resultados das DC com o IMC ( $r=-0.027$ e $\mathrm{p}=0.907)$, concluindo-se que o IAC tem uma maior aplicabilidade do que o IMC para indivíduos entre 19 e 49 anos, lembrando que 50\% dos avaliados eram considerados ativos, $20 \%$ muito ativos e $30 \%$ sedentários, classificados pelo IPAQ.

É valido ressaltar que quando confrontado o IMC e o IAC não se obteve uma correlação significativa $(\mathrm{r}=0,408, \mathrm{p}=0,073)$, assim, pode ser inferido que não há grande associação entre os 
métodos. A importância do uso de indicadores antropométricos indiretos tem como característica básica a facilidade de coleta dos dados e simplicidade na interpretação de seus resultados. O IAC ainda apresenta a vantagem de que pessoas com alto índice de massa magra não são classificadas como obesas, como ocorre no IMC. Recomenda-se para estudos futuros nessa área, que sejam feitas pesquisas com diversas populações e outras faixas etárias, para consolidação de tal fidedignidade do método.

\section{Referências}

BENEDETTI, T. R. B.; ANTUNES, P. C.; RODRIGUEZ-AÑEZ, C. R., MAZO, G. Z., PETROSKI, É. L. Reprodutibilidade e validade do Questionário Internacional de Atividade Física (IPAQ) em homens idosos. Revista Brasileira de Medicina do Esporte, Niterói, v. 13, n. 1, p. 11-16, jan./fev. 2007. Crossef

BERGMAN, R. N.; STEFANOVSKI, D.; BUCHANAN, T. A.; SUMNER, A. E.; REYNOLDS, J. C.; SEBRING, G.; XIANG, A. H.; WATANABE, R. M. A better index of body adiposity. Obesity Journal, Estados Unidos, v. 19, n. 5, p. 1083-1089, May 2011.

DELGADO, L. A. Avaliação da aptidão física: projeto de elaboração de sistema de informações. São Luis, 2004. Disponível em: <http://www.ebah.com.br/content/ABAAAAc90AG/avaliacaofisica-medidas-antropometricas-leonardo-arruda-delgado >. Acesso em: 05 mar. 2014.

ESCO, M. R. The accuracy of the body adiposity index for predicting body fat percentage in collegiate female athletes. The Journal of Strength \& Conditioning Research, v. 27, n. 27, p. 1679$1683,2012$.

FERNANDES, J. F. A prática da avaliação física. 2. ed. Rio de Janeiro: Shape, 2003.

FREEDMAN, D. S.; BLANCK, H. M.; DIETZ, W. H.; DASMAHAPATRA, P.; SRINIVASAN, S. R.; BERENSON, G. S. Is the body adiposity index (hip circumference/height 1.5 ) more strongly related to skinfold thicknesses and risk factor levels than is BMI? The Bogalusa Heart Study. British Journal of Nutrition, v. 109, n. 2, p. 338-345, Jan. 2013. erossef

GUEDES, D. P. Estudo da gordura corporal através da mensuração dos valores de densidade corporal e da espessura de dobras cutâneas em universitários. 1985. 277 f. Dissertação (Mestrado em Educação Física) - Universidade Federal de Santa Maria, Santa Maria.

JACKSON, A. S.; POLLOCK, M. L. Generalized equations for prediting body density of men. British Journal of Nutrition, v. 40, n. 3, p. 497-504, 1978. rossef

JANUÁRIO, R. S. B.; NASCIMENTO, M. A.; BARAZETTI, L. K.; REICHERT, F. F.; MANTOAN, J. P. B.; OLIVEIRA, A. R. Índice de massa corporal e dobras cutâneas como indicadores de obesidade em escolares de 8 a 10 anos. Revista Brasileira de Cineantropometria \& Desempenho Humano, v. 10, n. 3, p. 266-270, 2008.

JOHNSON, W.; CHUMLEA, W. C.; CZERWINSKI, S. A, DEMERATH, E. W Concordance of the recently published body adiposity index with measured body fat percent in European-American adults. Obesity, v. 20, n. 4, p. 900-903, Apr. 2012. Crossef

LEMACKS, J. L.; LIU, P. Y.; SHIN; H.; RALSTON, P. A.; ILICH, J. Z. Validation of body adiposity index as a measure of obesity in overweight and obese postmenopausal white women and its comparison with body mass index. Menopause, v. 19, n. 11, p. 1277-1279, Nov. 2012. rrossef 
LÓPEZ, A. A.; CESPEDES, M. L.; VICENTE, T.; TOMAS, M.; BENNASAR-VENY, M.; TAULER, P.; AGUILO, A. Body adiposity index utilization in a Spanish Mediterranean population: comparison with the body mass index. PLoS ONE, v. 7, n. 4, p. 1-7, 2012. crossef

MACHADO, A. F. Manual de avaliação física. São Paulo: Ícone, 2010.

MATSUDO, S. M.; ARAÚJO, T.; MATSUDO, V. R.; ANDRADE, D.; ANDRADE, E.; OLIVEIRA, L. C.; BRAGGION, G. Questionário Internacional de Atividade Física (IPAQ): estudo de validade e reprodutibilidade no Brasil. Revista Brasileira de Atividade Física \& Saúde, v. 6, n. 2, p. 5-18, 2001.

POLLOCK, M. L.; WILMORE, J. H. Exercícios na saúde e na doença: avaliação e prescrição para prevenção e reabilitação. Rio de Janeiro: Medsi, 1993.

POMPEU, F. A. M. S.; GABRIEL, D.; PENA, B. G.; RIBEIRO, P. Áreas de secção transversa do braço: implicações técnicas e aplicações para avaliação da composição corporal e da força dinâmica máxima. Revista Brasileira de Medicina do Esporte, v. 10, n. 3, p. 202-206, 2004. rrossef

SCHULZE, M. B.; THORAND, B.; FRITSCHE, A.; HARING, H. U.; SCHICK, F.; ZIERER, A.; RATHMAN, W.; KROGER, J.; PETERS, A.; BOEING, H.; STEFAN, N. Body adiposity index, body fat content and incidence of type 2 diabetes. Diabetologia, v. 55, n. 6, p. 1660-1667, June 2012. crossef

SUlINO, R. M.; FREITAS, W. Z.; SILVA, A. P.; RAMOS, L. E.; SILVA, E. Comparação entre o índice de adiposidade corporal e a avaliação da composição corporal através de medidas de dobras cutâneas. Coleção Pesquisa em Educação Física, v. 10, n. 1, p. 63-68, 2011.

SOUZA, W. C.; MASCARENHAS, L. P. G.; LIMA, V. A.; SOUZA, W. B.; GRZELCZAK, M. T.; TAJES JR, D.; BISHOP, J. E. Correlação entre o índice de adiposidade corporal (IAC) e índice de massa corporal (IMC) em atletas de futebol. In: III SEMINÁRIO INTEGRADO DE PESQUISA, ENSINO E EXTENSÃO, 2013, Porto União. Anais... Porto União: SIPEX, 2013. 